\title{
CATASTROPHIC DISRUPTION OF COMET ISON
}

\author{
Jacqueline V. Keane $^{1,2}$, Stefanie N. Milam ${ }^{3}$, Iain M. Coulson ${ }^{4}$, Jan T. Kleyna $^{1}$, Zdenek Sekanina ${ }^{5}$, Rainer Kracht ${ }^{6}$, \\ Timm-Emmanuel Riesen ${ }^{1,2}$, Karen J. Meech ${ }^{1,2}$, and Steven B. Charnley ${ }^{3}$ \\ ${ }^{1}$ Institute for Astronomy, University of Hawaii, 2680 Woodlawn Drive, Honolulu, HI 96822, USA; keane@ifa.hawaii.edu \\ ${ }^{2}$ NASA Astrobiology Institute, Honolulu, HI 96822, USA \\ ${ }^{3}$ Astrochemistry Laboratory, NASA GSFC, MS 690, Greenbelt, MD 20771, USA \\ ${ }^{4}$ Joint Astronomy Center, 660 North Aohoku Place, Hilo, HI 96720, USA \\ 5 Jet Propulsion Laboratory, California Institute of Technology, 4800 Oak Grove Drive, Pasadena, CA 91109, USA \\ ${ }^{6}$ Ostlandring 53, D-25335 Elmshorn, Schleswig-Holstein, Germany \\ Received 2016 March 2; revised 2016 July 29; accepted 2016 August 5; published 2016 November 9
}

\begin{abstract}
We report submillimeter 450 and $850 \mu \mathrm{m}$ dust continuum observations for comet $\mathrm{C} / 2012 \mathrm{~S} 1$ (ISON) obtained at heliocentric distances $0.31-0.08$ au prior to perihelion on 2013 November $28\left(r_{h}=0.0125 \mathrm{au}\right)$. These observations reveal a rapidly varying dust environment in which the dust emission was initially point-like. As ISON approached perihelion, the continuum emission became an elongated dust column spread out over as much as $60^{\prime \prime}\left(>10^{5} \mathrm{~km}\right)$ in the anti-solar direction. Deconvolution of the November $28.04850 \mu \mathrm{m}$ image reveals numerous distinct clumps consistent with the catastrophic disruption of comet ISON, producing $\sim 5.2 \times 10^{10} \mathrm{~kg}$ of submillimeter-sized dust. Orbital computations suggest that the SCUBA-2 emission peak coincides with the comet's residual nucleus.
\end{abstract}

Key words: comets: general - comets: individual (C/2012 S1 ISON) - methods: data analysis - methods: observational

\section{INTRODUCTION}

Comet C/2012 S1 (ISON; hereafter, comet ISON) was discovered on 2012 September 21 bright and active at 6.3 au (Nevski \& Novichonok 2012). With a perihelion distance of 0.0125 au $\left(\sim 2.7\right.$ solar radii $\left.\left(R_{\odot}\right)\right)$, it was predicted to become exceedingly bright, initiating many observing campaigns. The large number of optical observations from ground- and spacebased telescopes made it possible to have a complete view of the mass, size distribution, and evolution of the (sub)micronsized dust in the coma of comet ISON (Knight \& Battams 2014; Samarasinha et al. 2015). A comprehensive analysis of the light curve revealed that comet ISON's intrinsic brightness evolved in cycles of activity between heliocentric distances of 9.4 and $0.7 \mathrm{au}$, with the duration of the cycles progressively shortening (Sekanina \& Kracht 2014). After a brief quiescent period, comet ISON underwent significant outburst activity inside 0.65 au (Biver et al. 2013; Combi et al. 2014). In particular, the persistence of significantly elevated water production equating to a total surface area more than 10 times the original surface area of the nucleus was attributed to widespread fragmentation (Combi et al. 2014). The subsequent dramatic drop in gas-phase emissions lent support to this. Comet ISON's brightness briefly leveled off but then gradually increased through $0.17 \mathrm{au}$. Inside $0.16 \mathrm{au}$, comet ISON dramatically brightened, peaking in brightness at 0.051 au just prior to perihelion (Knight \& Battams 2014). After perihelion, all spacecraft data showed a steep and accelerating fading.

In recent years, spacecraft observations have revealed that the comae of comets contain significant populations of large particles with radii $r>1 \mathrm{~cm}$ that undergo fragmentation into smaller, millimeter-sized dust (Epifani et al. 2001; Hadamcik et al. 2013; Kelley et al. 2013). (Sub)Millimeter-wavelength observations are most sensitive to the thermal radiation emitted by millimeter-sized dust particles and have been shown to directly probe the grain mass contained within larger particles (Jewitt \& Luu 1990; Altenhoff et al. 1999; Jewitt \&
Matthews 1999; Bockelée-Morvan et al. 2010; Boissier et al. 2012). In this paper, we present single-dish Submillimeter Common-User Bolometer Array 2 (SCUBA-2) observations at $850 \mu \mathrm{m}$ of the dust continuum of comet ISON acquired over six days prior to perihelion on 2013 November 28.

\section{OBSERVATIONS}

Observations were undertaken on 2013 November 23.75-30.09 UT using the SCUBA-2 submillimeter camera on the James Clerk Maxwell Telescope (JCMT) located on Maunakea. The Gore-Tex membrane (opaque to visible photons) in front of the dish offered the unique opportunity to observe comet ISON's extremely close approach to the Sun. Imaging was achieved simultaneously at 450 and $850 \mu \mathrm{m}$. While atmospheric zenith opacities on the days involved were ideal, ranging between 0.08 nepers (at $225 \mathrm{GHz}$ on the first day) and 0.05 nepers (on the day of perihelion), the relatively low elevations $\left(30^{\circ}-45^{\circ}\right)$ and consequent high line-of-sight opacities limited the impact of the $450 \mu \mathrm{m}$ data. Additionally, thermal distortions of the dish during daylight hours disproportionally affected the quality of the $450 \mu \mathrm{m}$ signal.

Each of the focal planes of SCUBA-2 is populated with $\sim 5000$ bolometers, providing an instantaneous field of view (FOV) of $\sim 10^{\prime}$. To effectively account for the rapidly varying sky transmissions, the telescope scans rapidly around the target in one of a small number of patterns, depending upon the area to be mapped. In every pattern, each part of the target field is crossed in several directions by multiple bolometers, allowing for effective sky subtraction and image reconstruction. We assumed that ISON would be a compact target and adopted the daisy pattern with exposures of $\sim 30$ minutes, producing fairly uniform coverage in an area of $\sim 3^{\prime}$ around the target center and gradually diminishing the signal-to-noise ratio $(\mathrm{S} / \mathrm{N})$ beyond that. The major observational effect to account for during daytime is the degradation in shape of the primary mirror and the resulting impaired beam efficiency. Scans of astronomical 
Table 1

JCMT SCUBA-2 Observations of Comet C/2012 S1 (ISON) at $850 \mu \mathrm{m}$

\begin{tabular}{|c|c|c|c|c|c|c|c|c|c|c|}
\hline $\begin{array}{l}\text { UT Date } \\
2013 \text { November }\end{array}$ & $\begin{array}{c}R_{h} \\
(\mathrm{au})\end{array}$ & $\underset{(\mathrm{au})}{\Delta}$ & $\begin{array}{c}\alpha \\
(\operatorname{deg})\end{array}$ & $R_{\odot}$ & $\begin{array}{c}t_{\mathrm{P}} \\
(\mathrm{hr})\end{array}$ & $\begin{array}{c}T \\
(\mathrm{~K})\end{array}$ & $\begin{array}{c}\text { Peak }_{850 \mu \mathrm{m}} \\
(\mathrm{mJy} / \text { beam })\end{array}$ & $\begin{array}{c}S_{850 \mu \mathrm{m}} \\
(\mathrm{mJy})\end{array}$ & $\begin{array}{c}C_{e} \\
\left(10^{9} \mathrm{~m}^{2}\right)\end{array}$ & $\begin{array}{c}M \\
\left(10^{10} \mathrm{~kg}\right)\end{array}$ \\
\hline $23.75^{\mathrm{a}}$ & 0.311 & 0.867 & 99 & 66 & 121 & 499 & $170 \pm 8$ & $550 \pm 100$ & $4.9 \pm 0.7$ & $8.9 \pm 1.3$ \\
\hline 26.84 & 0.159 & 0.920 & 85 & 34 & 47 & 697 & $60 \pm 5$ & $330 \pm 82$ & $2.3 \pm 0.5$ & $4.2 \pm 1.0$ \\
\hline 26.86 & 0.159 & 0.923 & 85 & 34 & 46 & 700 & $60 \pm 6$ & $400 \pm 97$ & $2.8 \pm 0.6$ & $5.1 \pm 1.2$ \\
\hline 26.92 & 0.154 & 0.921 & 84 & 33 & 44 & 708 & $48 \pm 6$ & $230 \pm 77$ & $1.6 \pm 0.5$ & $2.9 \pm 0.9$ \\
\hline 26.96 & 0.152 & 0.922 & 84 & 33 & 43 & 712 & $54 \pm 6$ & $310 \pm 86$ & $2.1 \pm 0.6$ & $3.9 \pm 1.1$ \\
\hline 27.82 & 0.096 & 0.952 & 75 & 21 & 22 & 897 & $63 \pm 6$ & $410 \pm 92$ & $2.4 \pm 0.5$ & $4.4 \pm 0.9$ \\
\hline 28.04 & 0.080 & 0.961 & 72 & 17 & 17 & 982 & $97 \pm 6$ & $610 \pm 111$ & $3.3 \pm 0.6$ & $6.1 \pm 1.1$ \\
\hline
\end{tabular}

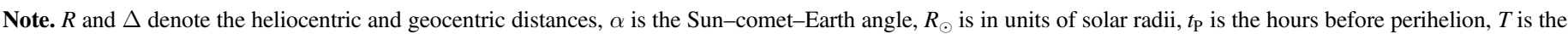

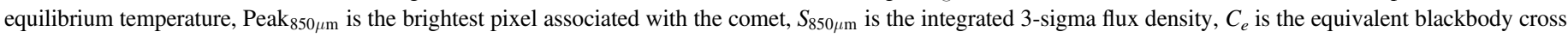
section, and $M$ is the dust mass within the projected area.

${ }^{\mathrm{a}}$ The date of the only unambiguous detection of comet ISON at $450 \mu \mathrm{m}: \frac{S_{450}}{S_{850}}=2.76 \pm 0.2$.

standards were taken regularly to confirm the instrument performance and to convert instrumental units $(\mathrm{pW})$ to energy units (jansky; Dempsey et al. 2013). Our observations were taken before mid-day, when efficiency degradation was measurable but not debilitating.

Pointing and non-sidereal guiding are controlled by the Telescope Control Software (TCS), which uses seven primary orbital elements ingested from JPL/Horizons. However, if the target passes a point in close proximity to a major body, as in comet ISON's approach to the Sun, the TCS struggles to accurately interpolate the elements. This results in apparent pointing errors amounting to $\sim 40^{\prime \prime}$ in the most extreme case. Nonetheless, the emissions from comet ISON always lie in well-exposed parts of the maps, and such displacements are not enough to cause inconsistencies in data reduction (see Section 3) and result merely in lower signal-to-noise ratios.

SCUBA-2 observations were reduced using the Starlink SMURF program within the Starlink Analysis and Reduction Software package (Chapin et al. 2013). The time-streams of signals from all bolometers are used to define "common-mode" variations due to the sky background. After subtraction of this "common-mode" signal, the residual signal from each bolometer is used to reconstruct the astronomical signal from the point on the sky that was viewed at each moment of the scan. This astronomical map is subtracted from the original data, and a new estimate of the common mode is made. The process iterates until the change between iterations becomes insignificant. Scan rates are $50^{\prime \prime}$ per second during daisies, and data sample rates are $200 \mathrm{~Hz}$, or once per 0.25 . The JCMT beam size at $850 \mu \mathrm{m}$ is $14^{\prime \prime}$ so point sources are well-sampled. Not knowing in advance the morphology or location of comet ISON during each observation, we reduced the data using a customized set of reduction parameters known as dimmconfig_jsa_generic.lis. Source pixels were taken to be those with a $\mathrm{S} / \mathrm{N}>3.0$, and during all iterations atmospheric correction was applied, and data were filtered to remove any features on scales larger than $200^{\prime \prime}$. The results presented here indicate no inadvertent suppression of real astronomical signals.

\section{RESULTS}

The submillimeter observations are summarized in Table 1 and Figures 1 and 2. Comet ISON, shown in Figure 1, was first successfully detected at both 450 and $850 \mu \mathrm{m}$ on November 23.75. The $3 \sigma$ detection of comet ISON at $450 \mu \mathrm{m}$ on November 23.75 is the only unambiguous detection, and unless otherwise noted, the remainder of the discussion focusses on the $850 \mu \mathrm{m}$ measurements. Figure 1 shows that comet ISON was offset from the central map position but was still well within the FOV. The continuum emission on November 23.75 is broader than a point source, with FWHMs of $26^{\prime \prime}(450 \mu \mathrm{m})$ and $23^{\prime \prime}(850 \mu \mathrm{m})$ and with the axis of greater breadth at a position angle of $103^{\circ}, \mathrm{E}$ of $\mathrm{N}$. The emission is also slightly asymmetric, with slight extension in the anti-solar direction. Comet ISON is offset (predominately in right ascension) from the expected JPL \#53 position by $\sim 4^{\prime \prime}$, denoted by the solid cross in each image. This offset from the expected position was first noted by Cordiner et al. (2014) in ALMA data taken on November 17 and later by Bonev et al. (2014) in IRTF/CSHELL observations on November 17-22. As comet ISON approached perihelion, this systematic offset became more pronounced (Figure 2).

The morphology of the continuum emission also changed dramatically. A coarse comparison suggests that in 5 days the morphology changed from a tightly bound slightly asymmetric sphere to an elongated dust column that was less tightly bound and spread out over as much as $60^{\prime \prime}\left(>10^{5} \mathrm{~km}\right)$ in the anti-solar direction.

Changes in comet ISON's orbit and solar vectors during the observing window were primarily on the plane of the sky and not in the line of sight. Therefore, the changes in morphology are not directly attributable to line-of-sight effects. As comet ISON neared perihelion, the central body disintegrated rapidly, its orbital motion deviating from that prescribed by gravitational law at a rate much steeper than anticipated by the standard formalism for nongravitational perturbations of cometary motions, driven by momentum transfer from the sublimation of water ice (Marsden et al. 1973). This motion was used to derive the JPL Horizons \#53 set of orbital elements. Realizing that the motion of ISON at these small heliocentric distances depends on the shrinking size of its nucleus and is strongly affected by the sublimation not only of water ice but also of species much more refractory, Sekanina \& Kracht (2014) introduced major modifications to the standard nongravitational law and successfully linked $\sim 560$ groundbased observations made between October 31 and November 22 with 126 observations by STEREO-B on November 26.3-28.5 UT. The orbital solution by Sekanina and Kracht, shown as dashed crosses in Figures 2 and 4, fits our SCUBA-2 $850 \mu \mathrm{m}$ images much better than the JPL \#53 orbit, suggesting 

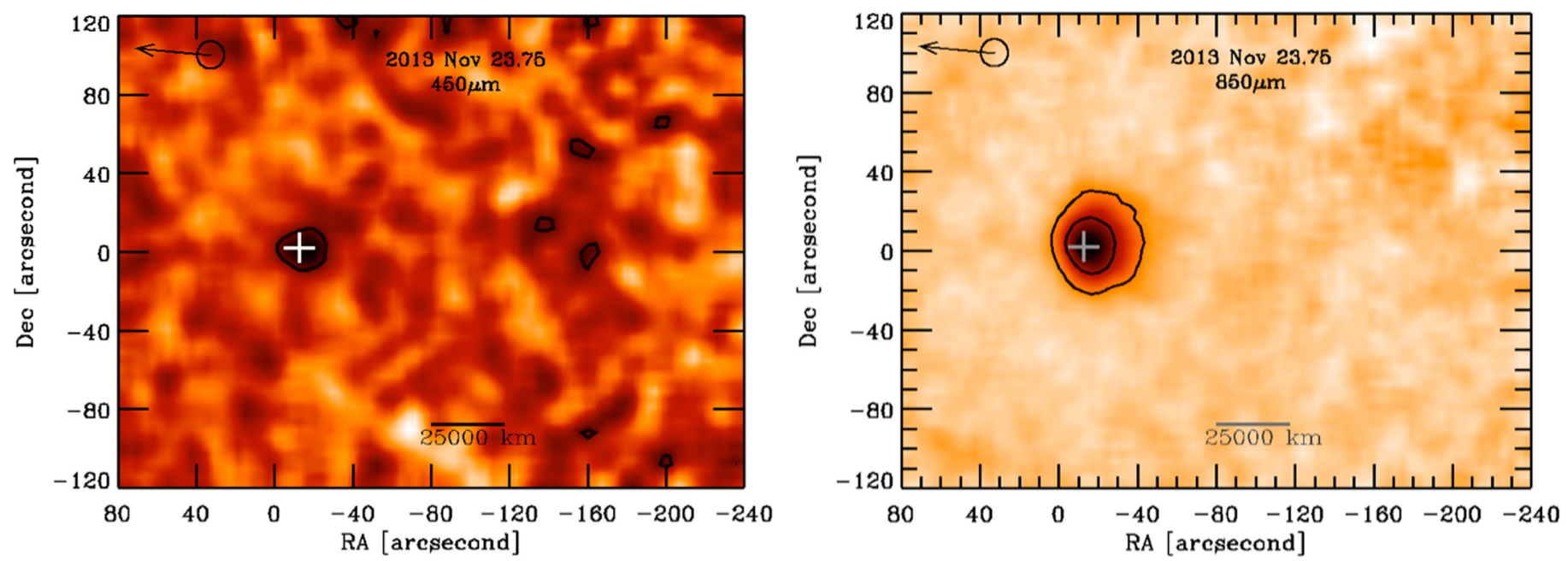

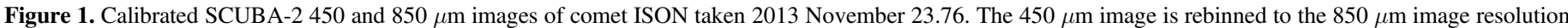

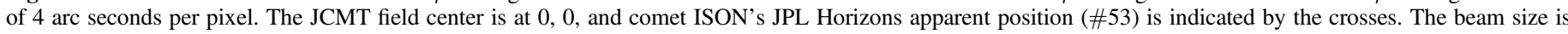

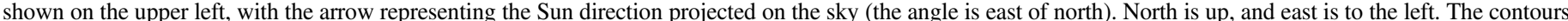

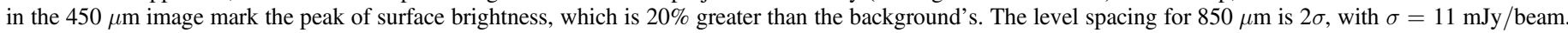
Each image represents $30 \mathrm{~min}$ of integration.

that the emission peak coincided with the residual nucleus measured in the STEREO-B images.

The FellWalker algorithm (Berry 2015) was used to independently determine clumps of emission in the maps, from which flux densities $\left(S_{850 \mu \mathrm{m}}\right)$ were extracted. Peak pixel brightnesses faded as the continuum spread out in the anti-solar direction, with a statistically significant rise in peak intensity prior to perihelion. And while the integrated flux densities exhibited a similar pattern, there was a marked steady increase in flux density by November 28.04. The rapid variability seen in the November 26 images cannot be attributed to any noncometary explanations, suggesting the submillimeter continuum of comet ISON varied over short timescales.

\subsection{Dust Mass and Mass-Loss Rate}

Little is known on the size distributions, optical properties, and kinematics of large cometary dust grains. Coupled with the dynamically varying dust environment of comet ISON and the dearth of simultaneous supporting observations, a timedependent analysis of the dust thermal emission is beyond the scope of this work. However, assuming the dust properties do not vary in the beam, $850 \mu \mathrm{m}$ brightness measurements are converted to emitting surface areas and dust masses using the simple approximation methods first introduced in Jewitt \& Luu (1990) and Jewitt \& Matthews (1999). The surface emission area $\left(C_{e}\right)$ is given by $S \times \Delta^{2} /\left(2 k T / \lambda^{2}\right)$, where $S$ is the integrated $850 \mu \mathrm{m}$ brightness given in Table $1, k_{\lambda}$ is the Boltzmann constant, $\lambda$ is the wavelength, and $\Delta$ is the geocentric distance. At heliocentric distances $r_{h}$ (au), the temperature of the dust grains, $T$, can be approximated by $278 \mathrm{~K} \times r_{h}^{-0.5}$ (cf. Jewitt \& Luu 1990). The dust mass is then given by $C_{e} / \kappa_{\lambda}$, where $\kappa$ is the dust opacity at $850 \mu \mathrm{m}$. The resulting emitting areas and dust masses are listed in Table 1, assuming a dust opacity of $0.055 \mathrm{~m}^{2} \mathrm{~kg}^{-1}$, which is analogous to the opacity of moderately porous grains (cf. Boissier et al. 2012). Though sample opacities for millimeter-sized grains of varying porosity, ice/silicate mixture, and size distribution have been published (Jewitt \& Luu 1990; Boissier et al. 2012), the nature of the millimeter grains cannot be determined with these observations. Dust masses could be significantly higher if the millimeter-sized dust grains are very porous, containing little ice. As noted earlier, $450 \mu \mathrm{m}$ emission was detected simultaneously with $850 \mu \mathrm{m}$ on November 23 . With an integrated intensity of $1.52 \mathrm{Jy}$, this gives a spectral index $(\beta)$ between 450 and $850 \mu \mathrm{m}$ of $\sim 0.35$, consistent with millimeter-sized grains (Pollack et al. 1994). Though a number of mid-infrared imaging observations were made in 2013 October in an attempt to constrain the grain size distribution (Ootsubo et al. 2013; Wooden et al. 2014), due to the rapid variability of comet ISON it is not realistic to devise a spectral energy distribution with such disparate data sets. The $S_{450} / S_{850}$ ratio is less than expected for a Rayleigh-Jeans blackbody. This may be indirect evidence for a steeper grain size distribution possibly due to either a varying grain size distribution or different opacities at $450 \mu \mathrm{m}$ and $850 \mu \mathrm{m}$.

Adopting a nucleus radius of $R_{\mathrm{N}}=500 \mathrm{~m}$ (consistent with the findings of Delamere et al. 2013; Combi et al. 2014; Lamy et al. 2014) and a bulk nuclear density of $470 \mathrm{~kg} \mathrm{~m}^{-3}$ (consistent with the mean bulk density of comet 9P (Thomas et al. 2013) and comet 67P (Sierks et al. 2015)) implies a nucleus of mass of $\sim 2.5 \times 10^{11} \mathrm{~kg}$. The millimeter-sized dust emitting at $850 \mu \mathrm{m}$ then accounts for $\sim 11 \%-34 \%$ of this mass. The dust mass production rate is determined by dividing the dust mass by the time $\left(t_{c}\right)$ it takes an ejected grain to leave the linear radius (in meters) of the projected beam $(P)$, where $t_{c}=P / V\left(r_{h}\right)$ (Jewitt \& Matthews 1999). Though morphological changes in optical images provide insight on the velocity of small dust, little is known on the kinematics of millimeter-sized dust. Epifani et al. (2001) showed millimeter-sized dust in the coma of $2 \mathrm{P} /$ Encke typically had velocities of $<10 \mathrm{~m} \mathrm{~s}^{-1}$ but reached velocities $>30 \mathrm{~m} \mathrm{~s}^{-1}$ approximately two weeks before perihelion. On the other hand, Boissier et al. (2012) found a population of millimeter-sized grains in the coma of $17 \mathrm{P} /$ Holmes with velocities of $50-100 \mathrm{~m} \mathrm{~s}^{-1}$ after a fragmentation event. Sekanina \& Chodas (2012) determined that as C/2013 W3 (Lovejoy) was disrupted shortly after perihelion, the millimeter-sized dust was released at velocities of up to $30 \mathrm{~m} \mathrm{~s}^{-1}$. Given this uncertainty in the velocity of millimeter- 

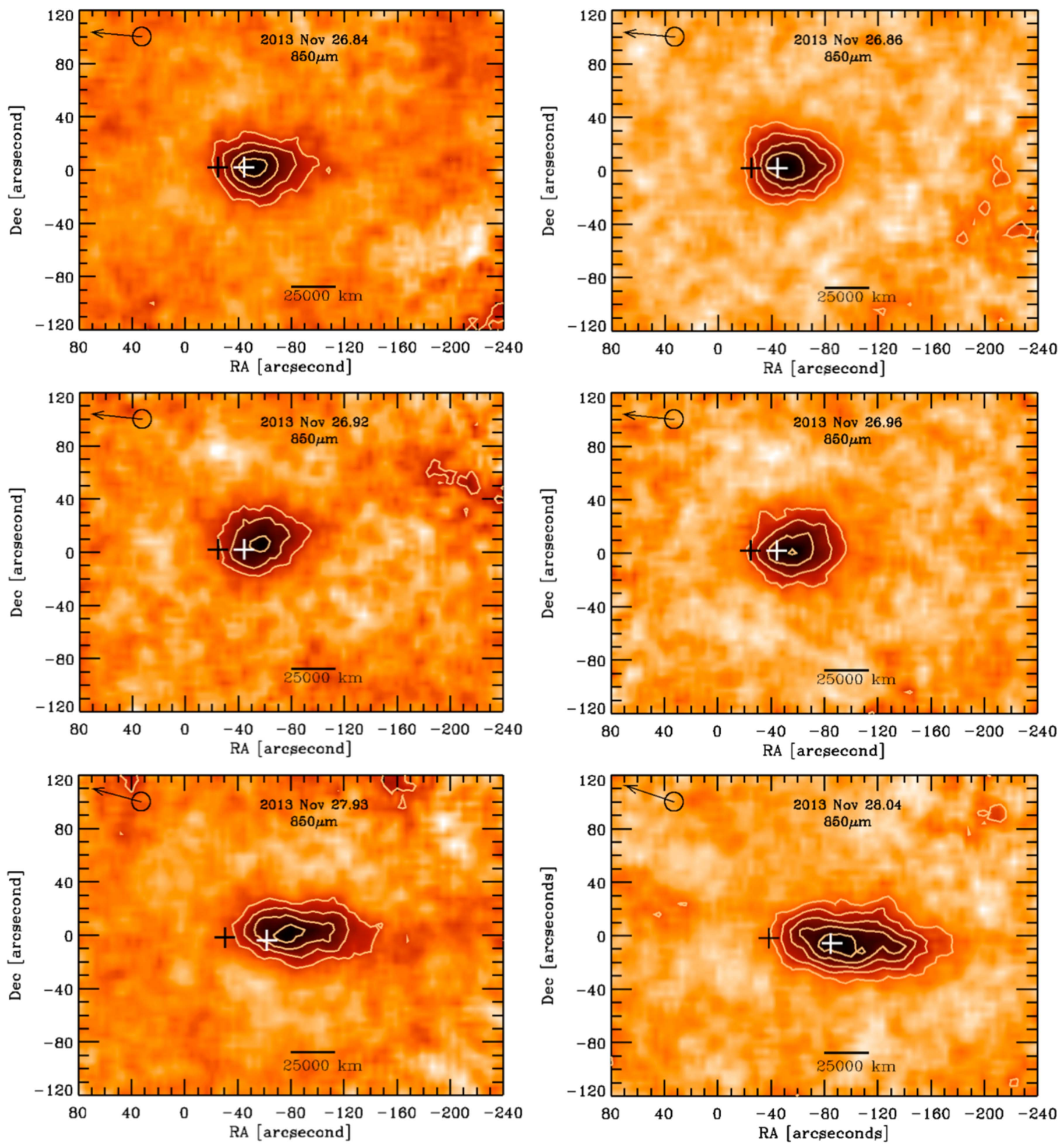

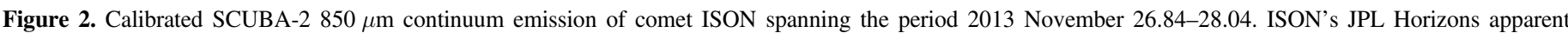

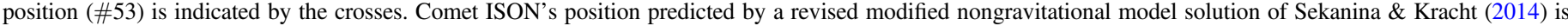
indicated by the white crosses. For additional figure description, see Figure 1.

sized grains, dust production rates were derived for different velocities $\left(V_{0}=25\right.$ and $\left.100 \mathrm{~m} \mathrm{~s}^{-1}\right)$ with $V\left(r_{h}\right)$ varying as a function of $V_{0} \times r_{h}^{-0.5}$, shown in Figure 3 . The average dust production rates are $6.4 \times 10^{5} \mathrm{~kg} \mathrm{~s}^{-1}$ and $2.8 \times 10^{6} \mathrm{~kg} \mathrm{~s}^{-1}$, respectively. Integrating over these observations (November 23.75-28.04) results in submillimeter dust mass fractions of $\sim 94 \%$ and $\sim 415 \%$ with respect to the total dust mass. While the comparably high ratio determined for millimeter-sized grains with velocities of $25 \mathrm{~m} \mathrm{~s}^{-1}$ is physically plausible, the fraction derived for velocities of $100 \mathrm{~m} \mathrm{~s}^{-1}$ is unphysical.

Moreno et al. (2014) modeled the evolution of the dust environment in the nucleus and coma of comet ISON as a function of heliocentric distance by analyzing a series of ground- and space-based optical images. An interesting result 


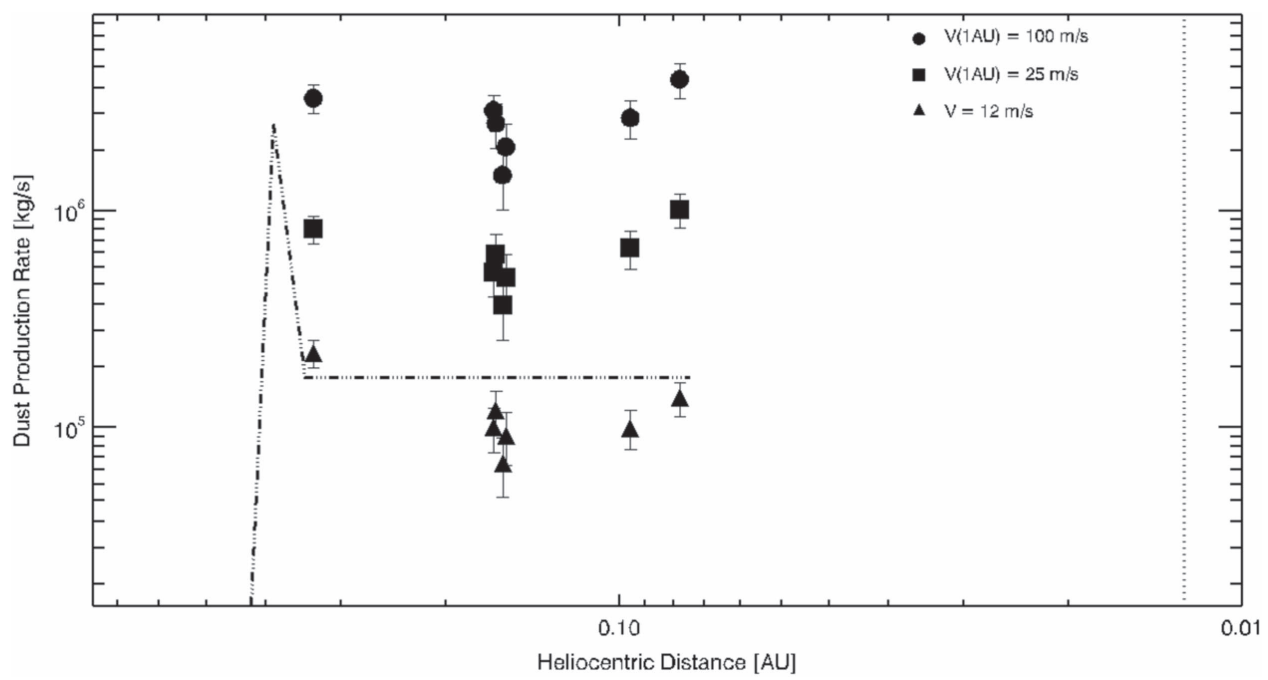

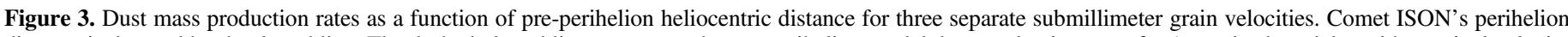

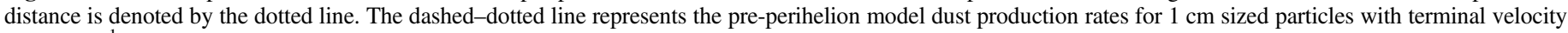
of $12 \mathrm{~m} \mathrm{~s}^{-1}$ from Moreno et al. (2014).

of this work is that the terminal velocity of $1 \mathrm{~cm}$ sized grains must be $\sim 10 \mathrm{~m} \mathrm{~s}^{-1}$ in order to accurately fit the observations. If we instead adopt a grain velocity of $12 \mathrm{~m} \mathrm{~s}^{-1}$, the average dust production rate becomes $1.4 \times 10^{5} \mathrm{~kg} \mathrm{~s}^{-1}$ (see Figure 3), resulting in $\sim 5.2 \times 10^{10} \mathrm{~kg}$ of dust produced from November 23.75-28.04. This would represent $\sim 20 \%$ of the total dust in a $500 \mathrm{~m}$ nucleus. Also shown in Figure 3 are the Moreno et al. dust production rates for $1 \mathrm{~cm}$ sized dust grains (dotted-dashed line); note that the line is truncated abruptly as the Moreno et al. analysis found that dust production ceased around November 28.07 . They infer that $\sim 2.3 \times 10^{11} \mathrm{~kg}$ of dust is needed to account for the dust in the tail seen from November 23-28. If that is the case, our observations show that the submillimeter dust accounts for $\sim 20 \%$ of the total dust produced between November 23 and 28. We note that dust mass fractions relate to a static non-decaying comet nucleus. If the nucleus was experiencing rapid erosion, then at 0.08 au the mass of millimeter-sized particles represented more than 100 percent of the nucleus mass.

\subsection{Fragmentation of Comet ISON}

The fact that comet ISON did not survive perihelion is not in dispute. Indeed, the central condensation that was consistently evident in $\mathrm{SOHO}$ pre-perihelion images abruptly disappeared on November 28.5 ( $~ 7 \mathrm{hr}$ before perihelion; Knight \& Battams 2014). The key question is, when did comet ISON begin to fatally fragment? Boehnhardt et al. (2013) reported finding coma wings in comet ISON on November 14 and November 16 $\left(r_{h} \approx 0.65\right.$ and $0.56 \mathrm{au}$ ). The presence of coma wings was interpreted as evidence for the existence of two or more subnuclei due to a recent split of comet ISON's nucleus. Coma wings have previously been detected in a number of comets confirmed to have undergone splitting events (Boehnhardt 2004, pp. 301-316). At closer heliocentric distances (0.53 and 0.34 au), Bonev et al. (2014) and DiSanti et al. (2016) used long-slit spectroscopy to probe the physical environment in the inner collisional coma of the comet. Contrary to the findings of Boehnhardt et al., the Bonev et al. and DiSanti et al. observations showed that the gas emission profiles of a number of species consistently exhibited a well- defined single peak, typical of an intact nucleus. Moreover, if ISON had fragmented at $\sim 0.66$ au with separation velocities of $\approx 2 \mathrm{~ms}^{-1}$, resulting in spatial separations of $\sim 500 \mathrm{~km}$, Bonev et al. would have detected double-peaked gas emission profiles. Though gas production rates varied dramatically between 0.53 and $0.35 \mathrm{au}$, there was no evidence for multiple peaks in the spatial profiles. It is important to note that if comet ISON had undergone a splitting event, multiple spatial peaks would not have been detected if the fragments had been short lived and/or had been oriented along the line of sight.

To explore whether there was evidence of discrete sources within the submillimeter continuum emission maps, we constructed a SCUBA-2 point-spread function (PSF) and applied a convolution kernel to the $850 \mu \mathrm{m}$ images. The deconvolution technique, which assumes the object is composed of small-scale point-like sources, iteratively fits and subtracts a synthetic Gaussian PSF beam to find discrete points with the highest absolute brightness. The main beam size at $850 \mu \mathrm{m}$ is $14^{\prime \prime}$, so we varied the FWHM of the Gaussian PSF from 3 ". 5 to 5 ". 9 to determine the robustness of the extracted structure. The retrieved structure did not vary with the PSF. It is important to note that the method of deconvolution used was not flux conserving; it merely served as a tool to search for discrete structures within the continuum emission. Figure 4 shows the resulting deconvolved images. Note that the four November 26 images were averaged and deconvolved as a single image. When this image was compared with the original $850 \mu \mathrm{m}$ continuum images (shown in Figures 1 and 2), the deconvolution technique found single, unresolved structures on November 23 and 26. However, in the case of November 27 the deconvolution technique suggested that there were at least two unresolved clumps. As time progressed, the deconvolved November 28 image revealed numerous point-like clumps emerging. The immediate conclusion that can be drawn from Figure 4 is that the bulk component(s) of comet ISON likely began to be disrupted near $0.15 \mathrm{au}$, which culminated in a cascading catastrophic fragmentation event between 0.096 and $0.08 \mathrm{au}$, as evidenced by the multiple discrete components in the expanding rubble pile. This is consistent with the findings of Moreno et al. (2014), who concluded that a significant mass- 

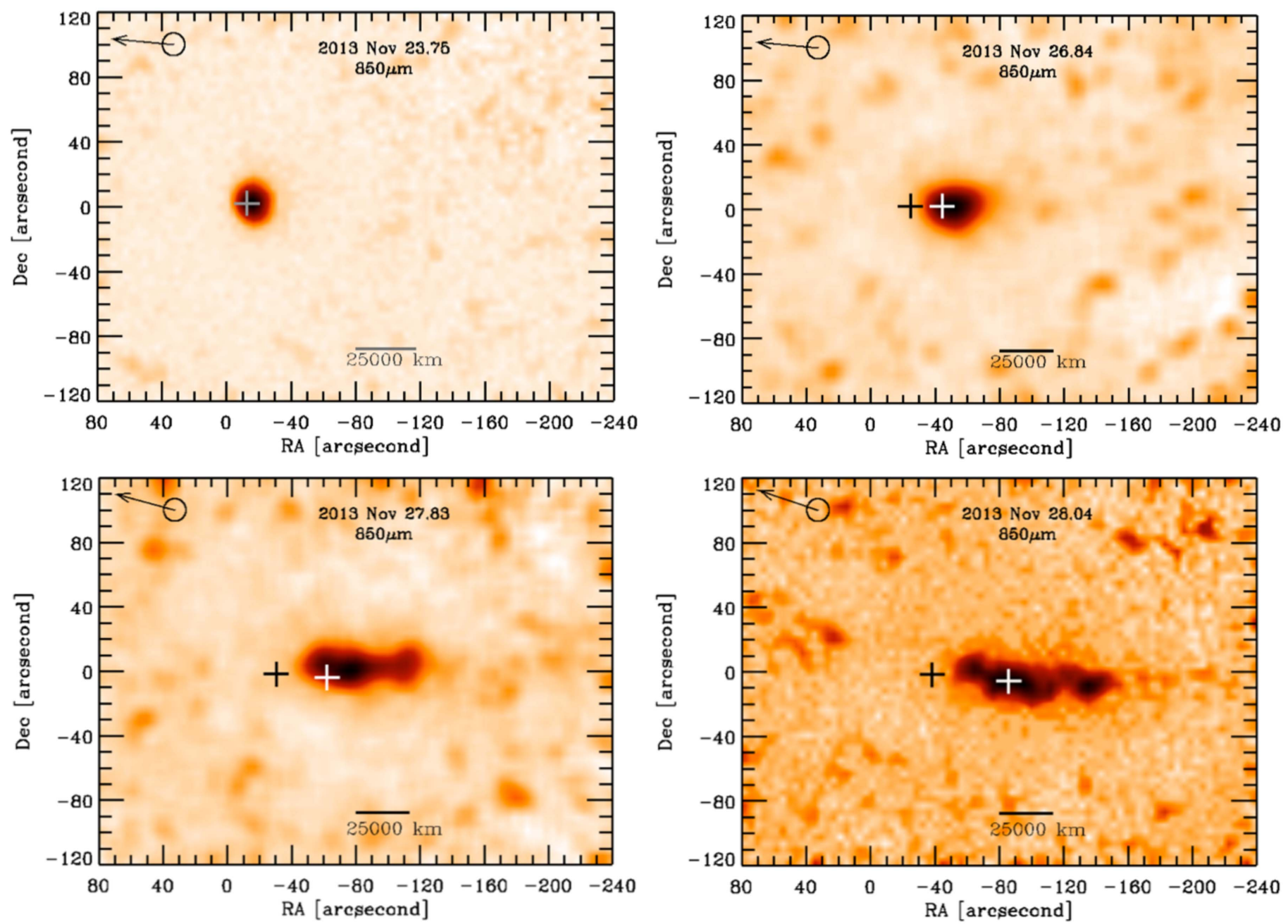

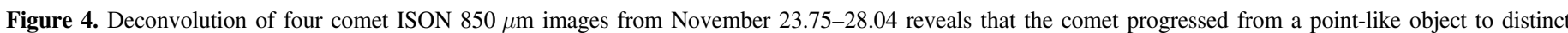
clumps. For additional figure description see Figure 1.

loss event started on November $23\left(r_{h} \sim 0.36 \mathrm{au}\right)$ and terminated in a cataclysmic fragmentation event on November 28.07 at 0.08 au (or $17 R_{\odot}$ ). We continued to monitor ISON with SCUBA-2 as it passed through perihelion and into the first few hours post-perihelion, but did not detect a statistically significant signal at $850 \mu \mathrm{m}$. Though the noise levels were stable ( $\sim 7 \mathrm{mJy}$ per pixel), the FellWalker algorithm failed to detect $850 \mu \mathrm{m}$ emission clumps with a 3-sigma upper limit of $<95 \mathrm{mJy}$. Our non-detection is consistent with the analysis of LASCO C3 images taken immediately prior to perihelion on November $28.62\left(r_{h}=0.026 \mathrm{au}\right)$, which revealed that most material (including any nucleus remnant) was rapidly vaporized or fragmented into very small dust grains after the catastrophic event (Moreno et al. 2014). Analysis of postperihelion $\mathrm{SOHO}$ LASCO $\mathrm{C} 3$ images suggests that the maximum size of the dust in the surviving debris cloud was likely to be $<50 \mu \mathrm{m}$, which is not detectable at $850 \mu \mathrm{m}$. As a final remark, we note that the 30 min images analyzed by the deconvolution technique are actually composed of $\sim 52$ exposures of $30 \mathrm{~s}$ each. In principle, this means that the data can be divided into more discrete time steps. However, a rigorous analysis is required to ensure a sufficient signal-tonoise ratio is preserved for point-like source detection.

\section{SUMMARY}

SCUBA-2, which operates on JCMT-EAO, observed the submillimeter dust continuum of comet ISON over five days, beginning on November 23 up to the 2013 November 28 perihelion. Deconvolution of the $850 \mu \mathrm{m}$ images shows that by November 28.04 numerous distinct point-like clumps had formed, consistent with comet ISON's catastrophic fragmentation. The clumps eventually vaporized. Orbital computations suggest that the SCUBA-2 emission peak coincides with the comet's residual nucleus. We expect that a more in-depth analysis on the position of each clump at finer time steps will yield significant insights into the outgassing perturbations and fragmentation event that led to the disruption of comet ISON. The limited role that these observations provide in determining grain properties and in constraining the size distribution shows that concurrent observations at visible, mid-IR, and submillimeter wavelengths are required to better probe the physical properties of the dust.

We are grateful to JCMT for their support during the observing campaign. The experience of telescope systems specialist Jim Hoge was particularly valuable. We also gratefully acknowledge the Time Allocation Committees of the JCMT - the UKTAG, the International TAC, and the 
University of Hawaii-for their support of long-term cometary science. The data were taken for programs m13bi07 and m13bh32a. JVK, JT Kleyna, and KJM acknowledge support through NSF Grant AST 1413736 and the NASA Astrobiology Institute under Cooperative Agreement NNA08DA77A. SNM acknowledges the NASA Planetary Astronomy Program.

Facility: JCMT.

\section{REFERENCES}

Altenhoff, W. J., Bieging, J. H., Butler, B., et al. 1999, A\&A, 348, 1020 Berry, D. S. 2015, A\&C, 10, 22

Biver, N., Agúndez, M., Santos-Sanz, P., et al. 2013, CBET, 3711, 2

Bockelée-Morvan, D., Hartogh, P., Crovisier, J., et al. 2010, A\&A, 518, L149 Boehnhardt, H. 2004, in Comets II, ed. M. C. Festou et al. (Tucson, AZ: Univ. Arizona Press), 301

Boehnhardt, H., Vincent, J. B., Chifu, C., et al. 2013, CBET, 3715

Boissier, J., Bockelée-Morvan, D., Biver, N., et al. 2012, A\&A, 542, A73

Bonev, B., DiSanti, M. D., Villanueva, G. L., et al. 2014, ApJL, 796, L6

Chapin, E. L., Berry, D. S., Gibb, A. G., et al. 2013, MNRAS, 430, 2545

Combi, M. R., Fougere, N., Mäkinen, J. T. T., et al. 2014, ApJL, 788, L7

Cordiner, M. A., Remijan, A. J., Boissier, J., et al. 2014, ApJL, 792, L2
Delamere, W. A., McEwen, A. S., Mattson, S., et al. 2013, CBET, 3720 Dempsey, J. T., Friberg, P., Jenness, T., et al. 2013, MNRAS, 430, 2534 DiSanti, M. A., Bonev, B., Gibb, E. L., et al. 2016, ApJ, 820, 34 Epifani, E., Colangeli, L., Fulle, M., et al. 2001, Icar, 149, 339

Hadamcik, E., Sen, A. K., Levasseur-Regourd, A. C., et al. 2013, Icar, 222, 634 Jewitt, D., \& Luu, J. 1990, ApJ, 365, 738

Jewitt, D., \& Matthews, H. 1999, AJ, 117, 1056

Kelley, M. S., Lindler, D. J., Bodewits, D., et al. 2013, Icar, 222, 634

Knight, M. A., \& Battams, K. 2014, ApJL, 782, L37

Lamy, P. L., Toth, I., Weaver, H. A., et al. 2014, ApJL, 794, L9

Marsden, B. G., Sekanina, Z., \& Yeomans, D. K. 1973, AJ, 78, 211

Moreno, F., Pozuelos, F., Aceituno, F., et al. 2014, ApJ, 791, 118

Nevski, B., \& Novichonok, A. 2012, CBET, 3238

Ootsubo, T., Watanabe, J., Honda, M., et al. 2013, in American Geophysical Union, Fall Meeting 2013, Abstract \# P24A-06

Pollack, J. B., Hollenbach, D., Beckwith, S., et al. 1994, ApJ, 421, 615

Samarasinha, N. H., Mueller, B. E. A., Knight, M. W., et al. 2015, P\&SS, 118,138

Sekanina, Z., \& Chodas, P. W. 2012, ApJ, 757, 127

Sekanina, Z., \& Kracht, R. 2014, arXiv:1404.5968

Sierks, H., Barbieri, C., Lamy, P. L., et al. 2015, Sci, 347

Thomas, P., A'Hearn, M., Belton, M. J. S., et al. 2013, Icar, 222, 453

Wooden, D. H., De Buizer, J. M., Kelley, M. S., et al. 2014, LPICo, 1777, 2906 